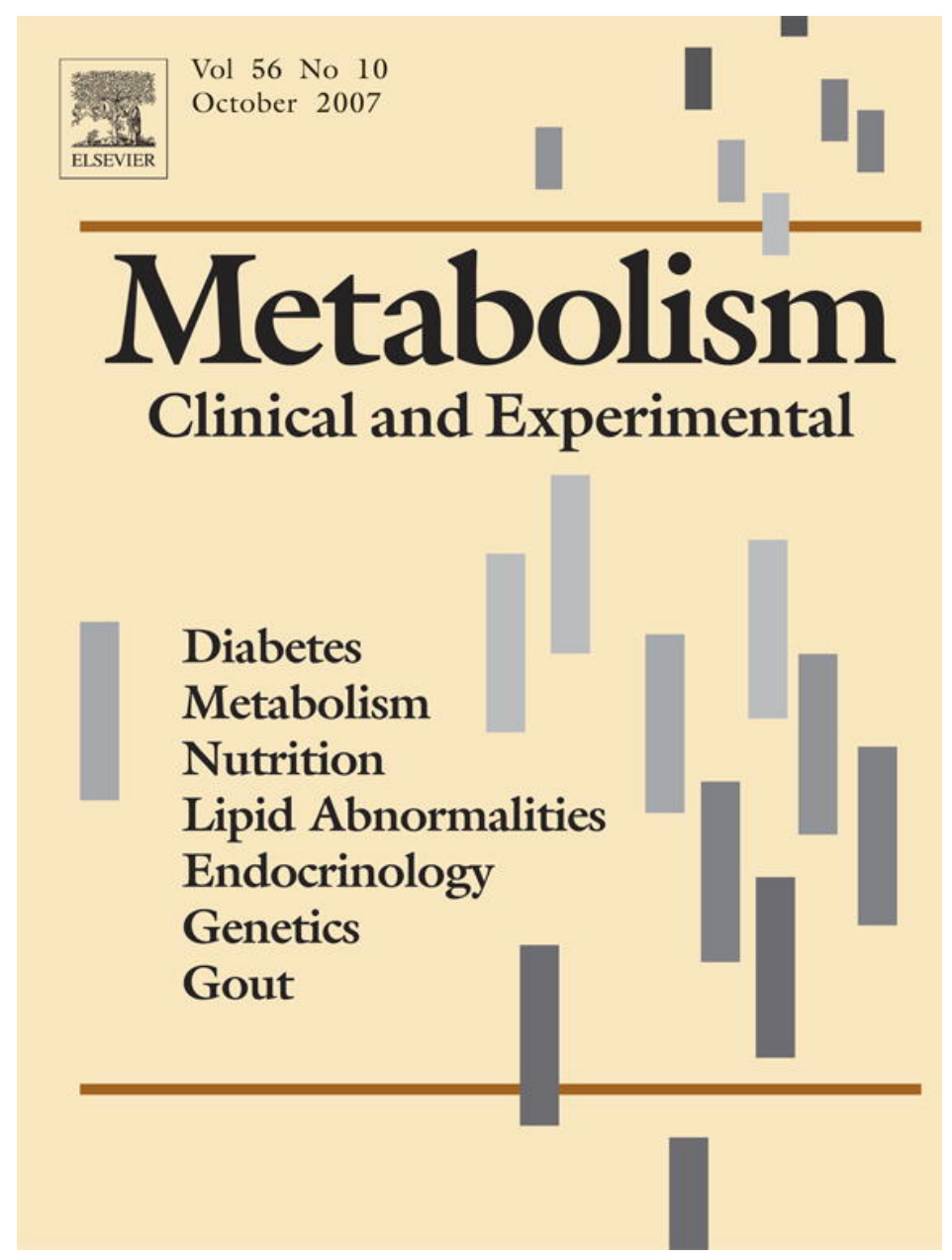

This article was published in an Elsevier journal. The attached copy

is furnished to the author for non-commercial research and education use, including for instruction at the author's institution, sharing with colleagues and providing to institution administration.

Other uses, including reproduction and distribution, or selling or licensing copies, or posting to personal, institutional or third party websites are prohibited.

In most cases authors are permitted to post their version of the article (e.g. in Word or Tex form) to their personal website or institutional repository. Authors requiring further information regarding Elsevier's archiving and manuscript policies are encouraged to visit: 


\title{
Diet-induced obesity in gravid rats engenders early hyperadiposity in the offspring
}

\author{
Silvia Caluwaerts ${ }^{\mathrm{a}}$, Suzan Lambin ${ }^{\mathrm{a}}$, Rita van Bree ${ }^{\mathrm{a}}$, Herman Peeters ${ }^{\mathrm{b}}$, \\ Ignace Vergote $^{\mathrm{a}}$, Johan Verhaeghe ${ }^{\mathrm{a}, *}$ \\ ${ }^{\mathrm{a}}$ Department of Obstetrics and Gynaecology, Katholieke Universiteit Leuven, 3000 Leuven, Belgium \\ ${ }^{\mathrm{b}}$ BMC Unit, Katholieke Universiteit Leuven, 3000 Leuven, Belgium \\ Received 2 October 2006; accepted 5 June 2007
}

\begin{abstract}
Exposure to a dysmetabolic in utero environment may be one of the mechanisms to explain why individuals with high birth weight are more likely to remain overweight. We explored this hypothesis in an animal model of diet-induced obesity (DIO). We studied adipose tissue development and glucose tolerance in the offspring of rat dams fed a diet rich in milk and sugar from early adulthood until day (d) 2 postpartum. This diet promoted body weight (BW) gain and was previously shown to produce insulin resistance and gestational glucose intolerance. The DIO offspring showed a higher BW in early life (between d7 and d35), with a maximum of 1 SD above the mean BW of controls; however, BW in DIO offspring after d35 was comparable with that of controls. Neonatal DIO offspring also showed larger fat depots, adipocyte hypertrophy $(P \leq .001)$, and more than 2-fold increased tumor necrosis factor $\alpha$ messenger RNA levels in subcutaneous adipose tissue $(P<.05)$. In addition, they displayed a higher peak glucose response to a glucose challenge $(P<.05)$. In postpubertal (d56) and adult (d98) offspring, we found differences in fat mass and distribution and glucose tolerance relating to the offspring's sex but not the maternal diet. In conclusion, DIO during pregnancy results in hyperadiposity and reduced glucose tolerance only in their neonatal/weanling but not postpubertal offspring. Future research should disclose whether these early-life effects are reactivated in conditions of heightened insulin resistance.

(C) 2007 Elsevier Inc. All rights reserved.
\end{abstract}

\section{Introduction}

The body mass index (BMI) of adolescents and young adults tracks with parental BMI and size at birth [1]. Both genetic and behavioral factors explain the robust correlation between parental and offspring BMI; in addition, the intrauterine environment may play a role. It is now fairly well documented that the risk of obesity is elevated at the low and high end of the birth weight spectrum [2]. At the high end, exposure in utero to a glucose-intolerant or diabetic environment is thought to reset metabolic pathways that facilitate fat accumulation and perturb glucose handling in postnatal life. Indeed, the BMI of children of mothers with gestational glucose intolerance was related to several

\footnotetext{
* Corresponding author. Tel.: +32 16 344212; fax: +32 16344205 .

E-mail address: johan.verhaeghe@uz.kuleuven.be (J. Verhaeghe).
}

measures of glycemic control during pregnancy (eg, postprandial glycemia, amniotic fluid insulin) $[3,4]$.

Studies in animal models may help unravel the contribution of the intrauterine environment to the risk of obesity. Holemans et al [5] studied the metabolic effects of dietinduced obesity (DIO) in gravid rats; the obesogenic diet was a highly palatable semisolid mixture of chow, condensed milk, and sugar [6]. This diet was commenced on day (d) 70 (early adulthood). The DIO rats gained more body weight (BW) and fat mass than did the rats fed the standard chow, and they were insulin resistant as shown by hyperinsulinemic clamps. Gestation had an additively negative effect on insulin sensitivity, leading to glucose intolerance in late gestation: the area under the glucose curve $\left(\mathrm{AUC}_{\text {glucose }}\right)$ was $41 \%$ higher in DIO dams than that in controls. Total litter weight (albeit not individual fetal weight) was increased by $25 \%$. Thus, an advantage of the DIO model compared with the frequently used streptozotocin-induced diabetes model is the absence of fetal hypotrophy $[7,8]$. 
In the current study, we used this DIO model to examine the effects of in utero exposure to an obesogenic diet on adipose tissue (AT) development and glucose tolerance at 3 ages (neonatal period, adolescence, and young adulthood). Adipose tissue development was assessed by weighing the various fat depots, measuring the number and size of adipocytes in 2 fat depots, and assaying the messenger RNA (mRNA) levels of peroxisome proliferator-activated receptor (PPAR) $\gamma$, a pivotal transcriptional regulator of adipocyte differentiation [9], and several adipokines-leptin, adiponectin, resistin, and tumor necrosis factor (TNF) $\alpha$-in the fat depots.

\section{Materials and methods}

\subsection{Animals, diets, and procedures}

The protocol was approved by the ethical committee for animal procedures. Thirty-six female (9 weeks old) and 6 male (12 weeks) Wistar rats were purchased from Charles River (Wilmington, MA) and housed in standard conditions. They had free access to tap water and a standard laboratory rat chow (Trouw, Gent, Belgium) (Table 1). One week after arrival, the female rats were randomly assigned to the control chow $(\mathrm{n}=18)$ or an experimental diet $(\mathrm{n}=$ 18), both available ad libitum. The experimental diet was prepared daily as a semisolid mixture of 33\% ground chow, sucrose, and 33\% unskimmed sweetened condensed milk (Nestlé, Vevey, Switzerland); its composition (analysis no. 678422 by LUFA-ITL, Kiel, Germany) is shown in Table 1. The mixture was presented into a purpose-designed plastic tray within the cage. Body weight was assessed weekly after introducing the diet. From 14 weeks on, the animals were mated overnight just before estrus, as detected in vaginal smears; the presence of spermatozoa the next morning was defined as $\mathrm{d} 0$ of gestation. Control rats and DIO dams were kept on their respective diets through gestation, and they were allowed to deliver. Litters were not culled or adjusted. Two days after delivery, DIO dams were switched back to the standard chow; this time point was chosen so as not to interfere with peripartum

Table 1

Composition of diets (in grams per kilogram original matter)

\begin{tabular}{lcc}
\hline & Control diet & Experimental (DIO) diet \\
\hline Moisture & 120 & 389 \\
Crude ash & 65 & 27 \\
Crude protein & 213 & 99 \\
Crude fat & 31 & 43 \\
Starch & 304 & 98 \\
Sugar & 70 & 254 \\
Organic rest & 149 & 76 \\
Crude fiber & 48 & 14 \\
Metabolizable energy (kJ/g) & 12.7 & 10.02 \\
Protein (energy \%) & 37.4 & 22.0 \\
Carbohydrate (energy \%) & 54.4 & 63.4 \\
Fat (energy \%) & 8.2 & 14.6
\end{tabular}

maternal behavior and the onset of lactation. The pups were weighed on $\mathrm{d} 2, \mathrm{~d} 7$, and weekly thereafter; they were weaned on $\mathrm{d} 21$, separated according to sex, and fed the standard chow throughout the experimental period. The offspring were assigned at random to the d14 to d21, d56, or $\mathrm{d} 98$ experiments. At weaning, the dams were euthanized with pentobarbital intraperitoneally (IP) (Santé Animale, Brussels, Belgium).

\subsubsection{Neonatal offspring}

The body composition was examined in d14, overnight fasted, and anesthetized $(0.024 \mathrm{mg} / \mathrm{kg}$ pentobarbital IP, to ensure immobility) pups with a Piximus 2 (Lunar, Madison, WI) densitometer, excluding the head area. After recovery, the pups were rejoined with their mothers. On $\mathrm{d} 21$, unanesthetized weanling rats received a glucose tolerance test (GTT) after an overnight fast (2 g/kg glucose, IP); blood samples (approximately $20 \mu \mathrm{L}$ ) were obtained before injection and after 20, 30, 60, 90, and 120 minutes by tail-snipping. Blood glucose was measured with a glucometer (Glucocard Memory 2 GT-1640, Menarini, Florence, Italy) that was calibrated before each GTT. The animals were euthanized thereafter. In a separate set of $\mathrm{d} 21$-anesthetized pups, a median laparotomy was performed and a blood sample was drawn from the aorta; the plasma was stored at $-80^{\circ} \mathrm{C}$. The perivisceral and inguinal white AT (WAT), nuchal WAT, and interscapular brown AT (BAT) were dissected and weighed. The WAT and BAT samples were snap-frozen in liquid nitrogen or immersed in Bouin fixative.

\subsubsection{Adolescent (d56) and adult (d98) offspring}

Glucose tolerance was examined by intravenous (IV) GTTs (1 g/kg glucose). On the eve, a catheter (Degania, Degania Bet, Israel) was inserted under anesthesia (ketamine $50 \mathrm{mg} / \mathrm{kg}$ [Parke-Davis, Zaventem, Belgium], xylazine $10 \mathrm{mg} / \mathrm{kg}$ [Bayer, Leverkusen, Germany], and atropine $0.25 \mathrm{mg} / \mathrm{kg}$ [Sterop, Brussels, Belgium], all IP) into the jugular vein, flushed with heparin in saline (0.1-0.2 mL), and tunneled to the back; water was freely available overnight. Blood glucose was measured on tail-prick samples before and after 5, 10, 15, 30, 60, and 90 minutes. At 10, 30, and 90 minutes, an additional $350 \mu \mathrm{L}$ of blood sample was collected by tail-snipping to measure plasma insulin; the animals were euthanized thereafter. In a separate set of overnight fasted, anesthetized rats $(0.06 \mathrm{mg} / \mathrm{kg}$ pentobarbital), body composition was determined by dual-energy x-ray absorptiometry (DXA) on a Hologic QDR-1000/W absorptiometer (Hologic, Vilvoorde, Belgium), followed by aortic blood and AT sampling as described.

\subsection{Plasma assays}

Insulin was measured by radioimmunoassay with rat insulin as the standard (Linco, St Charles, MO) and a guinea pig anti-rat insulin antiserum [5]. Leptin levels were determined by radioimmunoassay, with rat leptin as the 
standard and a guinea pig anti-rat leptin antiserum (Linco); the detection limit of the assay is $0.5 \mathrm{ng} / \mathrm{mL}$, and withinassay variation is $<5 \%$.

\subsection{Adipocyte size and number}

Paraffin-embedded $3-\mu \mathrm{m}$ sections were obtained with $50-\mu \mathrm{m}$ intersections and stained with hematoxylin-eosin. We evaluated 7 to 10 microscopic fields per animal, all on different sections, by computer-assisted image analysis (KS400, Zeiss, Jena, Germany).

Adipocyte number was assessed only in the $\mathrm{d} 56$ and $\mathrm{d} 98$ offspring. We used the method described by Rodbell [10], with slight modifications; in preliminary experiments, we tested various buffer volumes, collagenase concentrations, and incubation times to optimize adipocyte retrieval. Perigonadal and nuchal WAT (50-100 mg) was washed in saline, cut into small pieces, and transferred into a vial with $3 \mathrm{~mL}$ Krebs-Ringer bicarbonate (KRBA) buffer with 2\% bovine serum albumin, $3 \mathrm{mmol} / \mathrm{L}$ glucose $(\mathrm{pH} \mathrm{7.4)}$, and $1 \mathrm{mg} / \mathrm{mL}$ collagenase. The vial was incubated for 1 hour in a shaking water bath, manually shaken to liberate adipocytes, and centrifuged ( 1 minute at $400 g$ ); and the top layer was washed with $5 \mathrm{~mL} \mathrm{KRBA}$. This procedure was repeated. All procedures were at $37^{\circ} \mathrm{C}$. The adipocytes were resuspended in $1 \mathrm{~mL} \mathrm{KRBA}$, stained with trypan blue, and counted in a Fuchs-Rosenthal chamber.

\subsection{Quantitative real-time polymerase chain reaction}

RNA was extracted from homogenized AT using TriPure Reagent (Roche, Mannheim, Germany). Reverse transcription and real-time polymerase chain reaction (RT-PCR) were performed using an ABI 7000 sequence detector (Applied Biosystems, Lennik, Belgium); all materials were purchased from this manufacturer. One hundred nanograms of RNA was converted into $20 \mu \mathrm{L}$ of complementary DNA with TaqMan RT reagents with $2.5 \mu \mathrm{mol} / \mathrm{L}$ random hexamers. Real-time PCR was performed in duplicate, with the eukaryotic 18S ribosomal RNA as the housekeeping gene; validation experiments showed equal PCR efficiency for housekeeping and target genes. The target genes were as follows: leptin (sense primer AAGACCATTGTCACCAGGATCAA, antisense primer CCTCTGCCTGGCGGATAC, probe FAMTTCACACACGCAGTCG), adiponectin (sense primer TCCTGGAGAGAAGGGAGAGAAG, antisense primer TCTCCTGGGTCACCCTTAGG, probe FAM-ACGCAGGTGTTCTTG), resistin (sense primer GCCATCAGCAAGAAGATCAATCAAG, antisense primer GACCAGCAATGTAGGACAGTGT, probe FAM-TCCCTACTGCCAGCTGC), and assay-on-demand primers and probes for rat TNF- $\alpha$ (Rn 005620_m1), uncoupling protein 2 (UCP-2, Rn 00571166_m1), and PPAR- $\gamma$ (Rn 00440945_m1). Cycling conditions were $95^{\circ} \mathrm{C}$ for 15 seconds and $60^{\circ} \mathrm{C}$ for 1 minute (40 cycles). The data were obtained as cycle threshold $(\mathrm{Ct})$ values $(\Delta \mathrm{Ct}=\mathrm{Ct}$ target - Ct $18 \mathrm{~S}$ ribosomal RNA) and expressed as the n-fold change with the SEM calculated as $\left[2^{-\Delta \Delta \mathrm{Ct}} \cdot \ln 2 \cdot \operatorname{SD}(-\Delta \Delta \mathrm{Ct})\right] /$ (number of samples per group) $\left.^{1 / 2}\right]$ [11].

\subsection{Data analysis}

Data analysis was performed using GraphPad Prism software version 4.00 (San Diego, CA). Body weight in the $\mathrm{DIO}$ rats was calculated as an $\mathrm{SD}$ or $Z$ score (ie, $\mathrm{BW}$ - mean BW of control rats at that age). The homeostasis model assessment of insulin resistance (HOMA-IR) was calculated as fasting insulin (in microunits per milliliter) $\times$ fasting glucose (in millimoles per liter)/22.5. We calculated the $\mathrm{AUC}_{\text {glucose }}$ and $\mathrm{AUC}_{\text {insulin }}$ during the GTT and the glucose and insulin increment by subtracting basal from peak values; glucose and insulin clearance was calculated as the slope value of log-transformed values from the peak value to the last measurement.

The data of DIO and control dams were compared by unpaired $t$ tests. For the offspring data, we examined the respective effects of maternal diet and sex by general linear model (2-factor) analysis of variance (ANOVA). If an interaction between both factors was detected $(P<.05)$, we proceeded to 1-way ANOVA followed-if $P<.05$-by Bonferroni multiple-comparison test to examine specific intergroup differences. The data were expressed as means \pm SEM.

\section{Results}

\subsection{BW of dams and offspring}

The DIO rats weighed significantly more than the controls 1 week after diet randomization (Fig. 1), and the mean BW difference with control dams widened to approximately $32 \mathrm{~g}$ before mating. The DIO dams also weighed more than control dams during gestation, but gestational BW gain was comparable $(P=.50)$. The DIO dams were returned to the control chow on $\mathrm{d} 2$ postpartum; by $\mathrm{d} 7$, there was no longer a significant difference in BW between DIO and control dams.

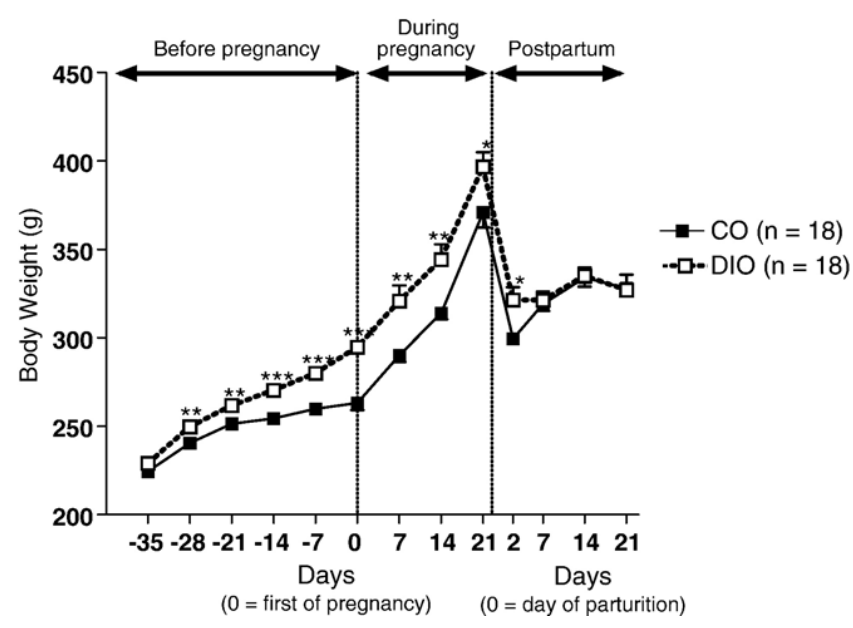

Fig. 1. Body weight in control (CO) and DIO dams. The experimental DIO diet was given from d70 until d2 postpartum. Data are means $\pm \mathrm{SEM}$. $* * * P<.001, * * P<.01, * P<.05$ ( $t$ tests). 


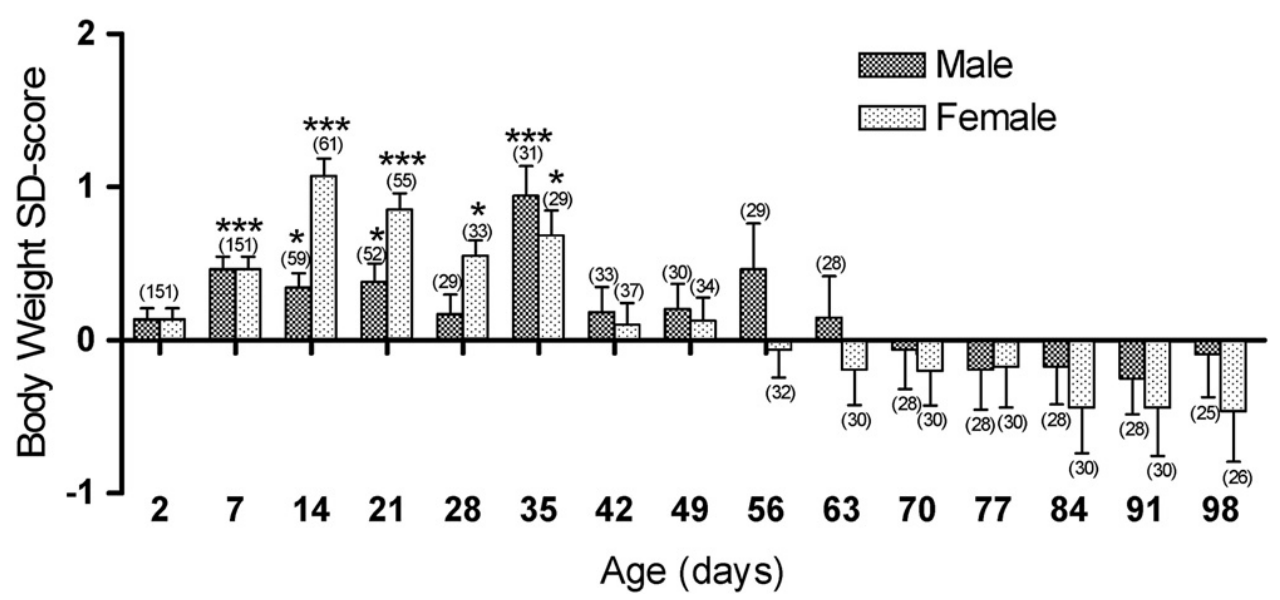

Fig. 2. Body weight SD score (BW - mean BW of controls at that age/SD of control BW) of DIO offspring. Data are means \pm SEM. $* * * P<.001, * P<.05$ ( $t$ test calculated on raw BW data). The maximum value was +0.94 SD on d35 in male DIO offspring and +1.07 SD on d14 in female DIO offspring.

Litter size was $10.1 \pm 0.56$ in DIO rats and $11.7 \pm 0.54$ in control rats $(P=.053)$. Although offspring $\mathrm{BW}$ was comparable on postnatal $\mathrm{d} 2(7.0 \pm 0.6 \mathrm{~g}$ in DIO offspring vs $6.9 \pm 0.7 \mathrm{~g}$ in controls, $P=.20$ ), BW of DIO offspring was higher than that in controls between postnatal $\mathrm{d} 7$ and $\mathrm{d} 35$; $\mathrm{BW}$ of control and DIO offspring was again comparable after d35. When expressed as SD scores, the maximum mean SD score was +1.07 for female DIO offspring on d14 and +0.94 for male DIO offspring on d35 (Fig. 2). Regression analysis of BW SD score with age showed negative slope values $(P=$ .008 and $P<.0001$ for male and female offspring, respectively), with a greater effect in female than in male rats $(P<.05)$.

\subsection{Body composition, fat mass, and plasma leptin in offspring}

The DXA-measured fat mass and fat percentage were elevated in d14 DIO offspring compared with those in controls (Table 2). In d21 DIO offspring, there was an excess of fat in the inguinal and nuchal regions; and there was more interscapular brown fat (Table 3). By d56, these diet-related differences were no longer observed, whereas sex-related differences became apparent. Indeed, male rats had a higher lean mass and a trend for a higher fat mass than female rats (DXA, Table 2), with more fat in the inguinal region, whereas female rats had more perirenal fat (Table 3). These observations were largely confirmed on d98: female rats now had a larger body fat percentage, and they maintained more perirenal fat than male rats.

Fasting plasma leptin was not different between DIO offspring and controls on d21, d56, or d98; but the levels were below the detection limit in most of the animals (data not shown).

\subsection{Adipocyte size and number}

Adipocyte size was higher in both perivisceral WAT and nuchal WAT of d21 DIO offspring compared with that in controls (Table 4). This hypertrophy had disappeared by d56; in fact, adipocyte size was smaller in nuchal WAT of DIO offspring than that of controls.

The number of adipocytes declined between $\mathrm{d} 56$ and $\mathrm{d} 98$ when expressed per gram fat $\left(23.0 \pm 0.9 \times 10^{6}\right.$ cells per $g$ fat

Table 2

Body composition, as measured by DXA, in control and DIO offspring

\begin{tabular}{|c|c|c|c|c|c|c|c|}
\hline & \multicolumn{2}{|c|}{ Control offspring } & \multicolumn{2}{|c|}{ DIO offspring } & \multicolumn{3}{|c|}{ 2-Factor ANOVA $(P)$} \\
\hline & Male & Female & Male & Female & Diet & Sex & Interaction \\
\hline D14 & $(\mathrm{n}=9)$ & $(\mathrm{n}=9)$ & $(\mathrm{n}=15)$ & $(\mathrm{n}=15)$ & & & \\
\hline Fat mass (g) & $2.2 \pm 0.2$ & $2.3 \pm 0.2$ & $2.6 \pm 0.1$ & $2.5 \pm 0.1$ & .048 & .85 & .39 \\
\hline Fat $\%$ & $11.3 \pm 0.5$ & $11.6 \pm 0.4$ & $12.5 \pm 0.5$ & $12.3 \pm 0.3$ & .038 & .95 & .63 \\
\hline D56 & $(\mathrm{n}=7)$ & $(\mathrm{n}=8)$ & $(n=7)$ & $(\mathrm{n}=7)$ & & & \\
\hline Fat mass $(\mathrm{g})$ & $17 \pm 2.3$ & $14.6 \pm 1.2$ & $17.9 \pm 1.6$ & $14.1 \pm 1.3$ & .90 & .069 & .66 \\
\hline Fat $\%$ & $7.2 \pm 0.9$ & $8.8 \pm 0.7$ & $7.5 \pm 0.8$ & $7.8 \pm 0.6$ & .63 & .23 & .39 \\
\hline D98 & $(\mathrm{n}=7)$ & $(\mathrm{n}=8)$ & $(\mathrm{n}=7)$ & $(\mathrm{n}=7)$ & & & \\
\hline Lean mass (g) & $340 \pm 10$ & $203 \pm 3$ & $328 \pm 7$ & $205 \pm 6$ & .51 & $<.0001$ & .31 \\
\hline Fat mass (g) & $39.3 \pm 4.6$ & $28.1 \pm 1.6$ & $28.8 \pm 2$ & $28.4 \pm 3.6$ & .13 & .081 & .11 \\
\hline Fat $\%$ & $10 \pm 1$ & $11.8 \pm 0.7$ & $7.8 \pm 0.5$ & $11.6 \pm 1.3$ & .24 & .0095 & .31 \\
\hline
\end{tabular}

Data are means \pm SEM. 
Table 3

Fat pad weights (in milligrams) in control and DIO offspring

\begin{tabular}{|c|c|c|c|c|c|c|c|}
\hline & \multicolumn{2}{|c|}{ Control offspring } & \multicolumn{2}{|c|}{ DIO offspring } & \multicolumn{3}{|c|}{ 2-Factor ANOVA $(P)$} \\
\hline & Male & Female & Male & Female & Diet & Sex & Interaction \\
\hline D21 & $(\mathrm{n}=11)$ & $(\mathrm{n}=11)$ & $(\mathrm{n}=12)$ & $(\mathrm{n}=13)$ & & & \\
\hline \multicolumn{8}{|l|}{ WAT subcutaneous } \\
\hline WAT inguinal & $133 \pm 14$ & $105 \pm 12$ & $188 \pm 8$ & $188 \pm 12$ & $<.0001$ & .28 & .27 \\
\hline WAT nuchal & $40 \pm 6$ & $36.5 \pm 6$ & $49.7 \pm 3$ & $47.7 \pm 4$ & .040 & .65 & .81 \\
\hline D56 & $(\mathrm{n}=7)$ & $(\mathrm{n}=8)$ & $(\mathrm{n}=6)$ & $(\mathrm{n}=7)$ & & & \\
\hline \multicolumn{8}{|l|}{ WAT perivisceral } \\
\hline Mesenteric & $439 \pm 76$ & $563 \pm 51$ & $547 \pm 55$ & $561 \pm 77$ & .43 & .31 & .42 \\
\hline Perirenal & $117 \pm 18$ & $413 \pm 33$ & $118 \pm 16$ & $454 \pm 97$ & .69 & $<.0001$ & .71 \\
\hline Perigonadal & $1486 \pm 166$ & $1318 \pm 111$ & $1523 \pm 115$ & $1156 \pm 205$ & .69 & .098 & .53 \\
\hline \multicolumn{8}{|l|}{ WAT subcutaneous } \\
\hline Inguinal & $1141 \pm 139$ & $730 \pm 41$ & $1100 \pm 98$ & $761 \pm 68$ & .95 & .0004 & .69 \\
\hline Nuchal & $261 \pm 31$ & $233 \pm 28$ & $255 \pm 19$ & $253 \pm 22$ & .79 & .57 & .61 \\
\hline D98 & $(\mathrm{n}=7)$ & $(\mathrm{n}=8)$ & $(\mathrm{n}=6)$ & $(\mathrm{n}=7)$ & & & \\
\hline \multicolumn{8}{|l|}{ WAT perivisceral } \\
\hline Mesenteric & $1764 \pm 254$ & $1372 \pm 72$ & $1387 \pm 113$ & $1403 \pm 86$ & .25 & .21 & .18 \\
\hline Perirenal & $606 \pm 100$ & $1580 \pm 174$ & $448 \pm 20$ & $1499 \pm 221$ & .45 & $<.0001$ & .81 \\
\hline Perigonadal & $4650 \pm 417$ & $3670 \pm 195$ & $3982 \pm 223$ & $3775 \pm 451$ & .43 & .10 & .28 \\
\hline \multicolumn{8}{|l|}{ WAT subcutaneous } \\
\hline Inguinal & $1993 \pm 57^{\mathrm{a}}$ & $1159 \pm 85^{\mathrm{b}}$ & $1646 \pm 80^{\mathrm{ac}}$ & $1321 \pm 116^{\mathrm{bc}}$ & .31 & $<.0001$ & $.0092 *$ \\
\hline Nuchal & $431 \pm 19$ & $397 \pm 26$ & $369 \pm 27$ & $412 \pm 47$ & .49 & .89 & .26 \\
\hline BAT & $204 \pm 27$ & $199 \pm 13$ & $177 \pm 15$ & $176 \pm 11$ & .16 & .85 & .88 \\
\hline
\end{tabular}

Data are means \pm SEM. Statistical analysis as explained in Materials and methods. Different superscripts indicate significant intergroup differences by Bonferroni post hoc test.

$* P<.0001$ for 1 -way ANOVA.

on d56 and $14.8 \pm 1.0 \times 10^{6}$ cells per $\mathrm{g}$ fat on $\mathrm{d} 98, P<.001$ ), but increased when expressed per total fat pad (18.4 $\pm 2.1 \times$ $10^{6}$ cells per fat pad on d56 and $27.5 \pm 3.5 \times 10^{6}$ cells per fat pad on $\mathrm{d} 98, P<.001)$. However, we observed no sex- or dietrelated effects in the adipocyte number on $\mathrm{d} 56$ or d98 (data not shown).

\subsection{Quantitative RT-PCR}

\subsubsection{Day 21}

Because neonatal DIO offspring showed hyperadiposity and adipocyte hypertrophy, we assessed the mRNA expression of leptin, adiponectin, resistin, TNF- $\alpha$, PPAR- $\gamma$, and UCP-2 in WAT (perivisceral, inguinal, and nuchal) and BAT.
We found no significant differences for leptin, adiponectin, resistin, and UCP-2 (data not shown). The PPAR- $\gamma$ expression was markedly lower in BAT of DIO offspring (2-factor ANOVA: $\operatorname{diet} P=.0004$, sex $P=.33$, interaction $P=.36$ ), but we found no significant differences in PPAR- $\gamma$ expression in any WAT sample (data not shown). In counterpart, TNF- $\alpha$ gene expression in inguinal and nuchal WAT was higher in DIO offspring than that in controls (Table 5).

\subsubsection{Day 56}

We quantified TNF- $\alpha$ mRNA levels to evaluate whether the $\mathrm{d} 21$ differences persisted. We found no significant differences except in the TNF- $\alpha$ expression in perigonadal WAT, which was increased in DIO offspring (Table 5).

Table 4

$\underline{\text { Surface area of AT cells (in square micrometers) in control and DIO offspring }}$

\begin{tabular}{|c|c|c|c|c|c|c|c|}
\hline & \multicolumn{2}{|c|}{ Control offspring } & \multicolumn{2}{|c|}{ DIO offspring } & \multicolumn{3}{|c|}{ 2-Factor ANOVA $(P)$} \\
\hline & Male & Female & Male & Female & Diet & Sex & Interaction \\
\hline D21 & $(\mathrm{n}=9)$ & $(\mathrm{n}=7)$ & $(\mathrm{n}=7)$ & $(\mathrm{n}=8)$ & & & \\
\hline WAT nuchal & $116 \pm 22$ & $98 \pm 7$ & $155 \pm 10$ & $177 \pm 9$ & .0008 & .87 & .21 \\
\hline \multicolumn{8}{|l|}{ D56 } \\
\hline WAT perivisceral & $461 \pm 101(n=4)$ & $475 \pm 60(n=6)$ & $449 \pm 22(n=5)$ & $435 \pm 67(n=5)$ & .68 & .99 & .84 \\
\hline D98 & $(\mathrm{n}=5)$ & $(\mathrm{n}=5)$ & $(n=6)$ & $(\mathrm{n}=8)$ & & & \\
\hline WAT perivisceral & $1980 \pm 135$ & $1988 \pm 219$ & $1851 \pm 158$ & $2036 \pm 256$ & .85 & .66 & .69 \\
\hline
\end{tabular}

Data are means \pm SEM. 
Table 5

Adipose tissue TNF- $\alpha$ mRNA levels in control and DIO offspring

\begin{tabular}{|c|c|c|c|c|c|c|c|}
\hline & \multicolumn{2}{|c|}{ Control offspring } & \multicolumn{2}{|c|}{ DIO offspring } & \multicolumn{3}{|c|}{ 2-Factor ANOVA $(P)$} \\
\hline & Male & Female & Male & Female & Diet & Sex & Interaction \\
\hline D21 & $(\mathrm{n}=9)$ & $(\mathrm{n}=7)$ & $(\mathrm{n}=7)$ & $(\mathrm{n}=8)$ & & & \\
\hline WAT perivisceral & $1 \pm 0.59$ & $1.07 \pm 0.73$ & $0.41 \pm 0.26$ & $0.54 \pm 0.25$ & .076 & .65 & .78 \\
\hline WAT inguinal & $1 \pm 0.69$ & $0.81 \pm 0.56$ & $2.30 \pm 1.79$ & $3.48 \pm 2.04$ & .027 & .92 & .52 \\
\hline WAT nuchal & $1 \pm 0.74$ & $0.14 \pm 0.10$ & $2.00 \pm 1.54$ & $2.14 \pm 1.60$ & .042 & .23 & .21 \\
\hline BAT & $1 \pm 0.26$ & $1.23 \pm 0.43$ & $1.07 \pm 0.27$ & $0.66 \pm 0.16$ & .13 & .44 & .067 \\
\hline WAT perigonadal & $1 \pm 0.28(\mathrm{n}=7)$ & $0.59 \pm 0.23(\mathrm{n}=8)$ & $1.88 \pm 0.64(\mathrm{n}=6)$ & $1.19 \pm 0.45(\mathrm{n}=7)$ & .034 & .11 & .91 \\
\hline WAT mesenteric & $1 \pm 0.45(\mathrm{n}=7)$ & $3.97 \pm 1.65(\mathrm{n}=8)$ & $3.46 \pm 1.47(\mathrm{n}=6)$ & $1.51 \pm 0.93(\mathrm{n}=7)$ & .71 & .47 & $.006^{\mathrm{a}}$ \\
\hline WAT inguinal & $1 \pm 0.57(\mathrm{n}=4)$ & $0.95 \pm 0.55(\mathrm{n}=6)$ & $0.93 \pm 0.47(\mathrm{n}=6)$ & $1.56 \pm 0.90(\mathrm{n}=6)$ & .61 & .56 & .49 \\
\hline WAT nuchal & $1 \pm 0.25(\mathrm{n}=5)$ & $4.26 \pm 2.69(n=7)$ & $1.36 \pm 0.46(n=6)$ & $1.45 \pm 0.40(n=6)$ & .37 & .08 & .11 \\
\hline BAT & $1 \pm 0.92(\mathrm{n}=5)$ & $1.03 \pm 0.71(n=6)$ & $0.41 \pm 0.29(\mathrm{n}=6)$ & $0.77 \pm 0.66(\mathrm{n}=5)$ & .24 & .51 & .54 \\
\hline
\end{tabular}

Data are means \pm SEM

a No significant differences after Bonferroni post hoc test.

\subsection{Glucose tolerance and insulin concentrations}

On d21, DIO offspring showed higher postchallenge glucose peak values than did controls (Table 6). On d56 and $\mathrm{d} 98$, there were no significant differences bet- ween DIO offspring and controls. Regarding sex-related differences, the $\mathrm{AUC}_{\text {glucose }}$ tended $(P=.089)$ to be higher in male than in female rats on $\mathrm{d} 21$, although this difference was highly significant on $\mathrm{d} 56$ but not $\mathrm{d} 98$. On $\mathrm{d} 98$, male rats had higher fasting insulin concentrations

Table 6

Glucose and insulin dynamics during GTT in control and DIO offspring

\begin{tabular}{|c|c|c|c|c|c|c|c|}
\hline & \multicolumn{2}{|c|}{ Control offspring } & \multicolumn{2}{|c|}{ DIO offspring } & \multicolumn{3}{|c|}{ 2-Factor ANOVA $(P)$} \\
\hline & Male & Female & Male & Female & Diet & Sex & Interaction \\
\hline D21 (IP GTT) & $(\mathrm{n}=8)$ & $(\mathrm{n}=8)$ & $(n=9)$ & $(n=7)$ & & & \\
\hline Fasting insulin & $48.2 \pm 25.7$ & $33.3 \pm 14.7$ & $23.2 \pm 9.1$ & $51.1 \pm 30.2$ & .90 & .76 & .27 \\
\hline HOMA-IR & $13.6 \pm 6.2(n=4)$ & $8.1 \pm 3.7(n=4)$ & $5.2 \pm 2(\mathrm{n}=5)$ & $5.9 \pm 3.4(\mathrm{n}=5)$ & .19 & .54 & .44 \\
\hline $\mathrm{AUC}_{\text {glucose }}$ & $10807 \pm 932$ & $8769 \pm 989$ & $12122 \pm 1152$ & $10599 \pm 955$ & .13 & .089 & .80 \\
\hline Peak glucose value (mg/dL) & $275 \pm 15$ & $290 \pm 17$ & $320 \pm 15$ & $306 \pm 9$ & .045 & .95 & .32 \\
\hline Glucose clearance $\left(10^{-3}\right.$ slope $)$ & $-3.6 \pm 0.3$ & $-4.2 \pm 0.4$ & $-4.5 \pm 0.2$ & $-3.7 \pm 0.2$ & 67 & .88 & $.037^{*}$ \\
\hline D56 (IV GTT) & $(\mathrm{n}=8)$ & $(\mathrm{n}=8)$ & $(n=7)$ & $(n=7)$ & & & \\
\hline Fasting insulin & $20.5 \pm 3.4$ & $20 \pm 2.8$ & $18.5 \pm 2.4$ & $19.1 \pm 3$ & 62 & .98 & .85 \\
\hline HOMA-IR & $3.8 \pm 0.7$ & $3.6 \pm 0.5$ & $3.3 \pm 0.5$ & $3.6 \pm 0.6$ & .73 & .88 & .68 \\
\hline AUC $_{\text {glucose }}$ & $6478 \pm 502$ & $5025 \pm 482$ & $6885 \pm 596$ & $4465 \pm 481$ & .88 & .0008 & .35 \\
\hline Peak glucose value (mg/dL) & $239 \pm 24$ & $199 \pm 12$ & $268 \pm 26$ & $229 \pm 15$ & .16 & .063 & .96 \\
\hline Glucose increase ( $\left.\Delta G_{\text {basal - peak }}\right)$ & $166 \pm 24$ & $127 \pm 11$ & $194 \pm 28$ & $152 \pm 15$ & .20 & .059 & .94 \\
\hline $\mathrm{AUC}_{\text {insulin }}$ & $1979 \pm 368$ & $1492 \pm 215$ & $1660 \pm 198$ & $1921 \pm 262$ & .84 & .68 & .19 \\
\hline Peak insulin value $(\mu \mathrm{U} / \mathrm{mL})$ & $106 \pm 21$ & $78 \pm 7$ & $93 \pm 11$ & $108 \pm 11$ & .55 & 65 & .15 \\
\hline Insulin increase $\left(\Delta I_{\text {basal }}\right.$ - peak $)$ & $85 \pm 19$ & $58 \pm 9$ & $74 \pm 12$ & $88 \pm 9$ & .49 & 67 & .13 \\
\hline Insulin clearance $\left(10^{-3}\right.$ slope $)$ & $-6.8 \pm 1$ & $-6.1 \pm 0.9$ & $-9.2 \pm 1.2$ & $-7.5 \pm 0.5$ & .059 & .20 & .58 \\
\hline D98 (IV GTT) & $(\mathrm{n}=8)$ & $(\mathrm{n}=8)$ & $(\mathrm{n}=8)$ & $(\mathrm{n}=8)$ & & & \\
\hline Fasting insulin & $44.4 \pm 4$ & $29.4 \pm 4.1$ & $50 \pm 7.7$ & $29.3 \pm 3.2$ & .57 & .001 & .56 \\
\hline HOMA-IR & $8.64 \pm 0.9$ & $5.5 \pm 0.7$ & $9.9 \pm 1.6$ & $5.2 \pm 0.5$ & .64 & .0006 & .43 \\
\hline $\mathrm{AUC}_{\text {glucose }}$ & $6822 \pm 807$ & $6340 \pm 518$ & $6943 \pm 1065$ & $6454 \pm 1018$ & .89 & .58 & .99 \\
\hline Peak glucose value (mg/dL) & $292 \pm 22$ & $260 \pm 32$ & $302 \pm 27$ & $272 \pm 36$ & .72 & .31 & .96 \\
\hline Glucose increase $\left(\Delta G_{\text {basal }- \text { peak }}\right)$ & $214 \pm 21$ & $184 \pm 31$ & $222 \pm 27$ & $391 \pm 35$ & .73 & .33 & .94 \\
\hline Glucose clearance $\left(10^{-3}\right.$ slope $)$ & $7.41 \pm 0.49$ & $6.9 \pm 0.62$ & $7.1 \pm 0.7$ & $6.5 \pm 0.06$ & .56 & .38 & .96 \\
\hline $\mathrm{AUC}_{\text {insulin }}$ & $2348 \pm 224$ & $1485 \pm 313$ & $2668 \pm 442$ & $2407 \pm 418$ & .096 & .13 & .41 \\
\hline Peak insulin value $(\mu \mathrm{U} / \mathrm{mL})$ & $144 \pm 10$ & $70 \pm 5$ & $153 \pm 22$ & $128 \pm 25$ & .07 & .0082 & .17 \\
\hline Insulin increase $\left(\Delta I_{\text {basal }}-\right.$ peak $)$ & $100 \pm 9^{\mathrm{a}}$ & $41 \pm 5^{\mathrm{b}}$ & $103 \pm 17^{\mathrm{a}}$ & $100 \pm 23^{\mathrm{ab}}$ & .050 & .054 & $.076^{* *}$ \\
\hline Insulin clearance $\left(10^{-3}\right.$ slope $)$ & $-6.1 \pm 0.5$ & $-2.3 \pm 3.4$ & $-5.2 \pm 0.5$ & $-5 \pm 0.8$ & .64 & .29 & .34 \\
\hline
\end{tabular}

Data are means \pm SEM. Statistical analysis as explained in Materials and methods. Different superscripts indicate significant intergroup differences by Bonferroni post hoc test.

* $P=.14$.

$* * P=.003$ for 1-way ANOVA 
and higher postchallenge insulin peak values than did female rats.

\section{Discussion}

The experimental diet rich in milk and sugar caused prompt and sustained weight gain, confirming our previous findings; this DIO model was also shown to provoke glucose intolerance at the end of gestation [5]. In the present study, we were interested in the short- and long-term metabolic effects of exposure in utero to such environment. Although $\mathrm{BW}$ on postnatal $\mathrm{d} 2$ was normal in DIO offspring, their BW was increased between $\mathrm{d} 7$ and $\mathrm{d} 35$, as was their fat mass and adipocyte size. These results support the concept that a dysmetabolic in utero environment affects AT development $[2,11]$. Rats contain only $1.1 \%$ fat tissue at birth compared with $16.1 \%$ in humans [12]. However, there are active proliferation and differentiation of preadipocytes in the perinatal period [13] that may well be modulated by the metabolic milieu, as shown in vitro $[14,15]$. Somewhat at odds with their adipocyte hypertrophy, normal plasma leptin in young DIO offspring was found. However, the leptin levels were very low; and in retrospect, it might have been preferable to measure leptin in nonfasting rather than fasting conditions or during the GTT.

The neonatal DIO offspring also showed reduced glucose tolerance (higher peak glucose value). We submit that this finding might be related to their adipocyte hypertrophy. Indeed, large adipocyte size in subcutaneous trunk fat was an independent predictor of type 2 diabetes mellitus in diabetesprone Pima Indians [16]; in mice, PPAR- $\gamma$ agonists prevented adipocyte hypertrophy and improved insulin sensitivity [9].

We documented a 2 - to 3 -fold increase in TNF- $\alpha$ mRNA levels in WAT of d21 DIO offspring. This finding confirms the increased TNF- $\alpha$ peptide expression and bioactivity in WAT of young rats fed a high-fat diet $[17,18]$. The TNF- $\alpha$ overexpression in AT of obese animals is primarily the result of macrophage infiltration [19]. Tumor necrosis factor $\alpha$ is believed to be one of the mediators that explain the link between adipocyte hypertrophy and insulin resistance (reviewed in [20]) and is likely an important adipocytokine in AT development. Indeed, TNF- $\alpha$ administration in gravid mice and rats caused overweight and hyperadiposity in their postpubertal female offspring [20,21].

The early relative overweight among DIO offspring was reversible, with a negative slope between BW SD score and age (Fig. 2). In addition, both the relative adipocyte hypertrophy and reduced glucose tolerance were no longer observed among postpubertal DIO offspring. The only difference we observed in d56 offspring was increased TNF- $\alpha$ mRNA levels in perigonadal WAT (Table 5). Thus, hyperadiposity conferred by a DIO environment in utero is more prominent at an early age. Our results echo findings obtained in humans: higher postchallenge glucose values in late pregnancy among nondiabetic Pima Indian mothers predicted a higher BMI and waist circumference in their children, but this association was strongest at a young offspring age [22]. We acknowledge limitations in the adipose cell number methodology. Collagenase may cause adipocyte rupture, especially of larger adipocytes. However, freshly siliconized glassware was used; and centrifugation steps were minimized [23]. Definitive conclusions on AT cellularity in DIO offspring await confirmation using complementary techniques [24].

Several recent studies have revealed long-term effects of specific diets during gestation in the rat. For example, the young adult offspring of dams fed a diet rich in $\omega-6$ polyunsaturated fat during pregnancy showed an increased body fat percentage and a higher insulin peak value in response to a glucose challenge [25]. Adult animals exposed in utero to a lard-based high-fat diet also demonstrated hyperadiposity (male animals only) and hyperinsulinemia [26]. The type of diet is of great importance because some high-fat or cafeteria diets had a deleterious effect on pregnancy outcome (litter size, birth weight, neonatal weight gain) [27-29]. The advantages of our DIO model include prompt weight gain after diet exposure, glucose intolerance in late gestation [5], and normal pregnancy characteristics. In the current study, we were unable to measure the food intake of DIO rats accurately owing to spilling of the semisolid diet within the cages. However, it is known that sweet-tasting, semisolid diets are particularly likely to promote hyperphagia and obesity in rats, more so than high-fat diets and pelleted/powdered diets [30]. Although the experimental diet contained relatively less protein than the standard diet, lowprotein diets during rat gestation have not been reported to cause maternal glucose intolerance or accelerated weight gain in the pups [31].

In the present study, the experimental diet was given until $\mathrm{d} 2$ postpartum. We did so to examine specifically the effect of in utero exposure and to avoid consumption of the diet by the neonates in week 3 . In addition, the rat neonatal period (d2-d20) is accompanied by remodeling of the islets of Langerhans [32]. It is clear that the long-term metabolic effects of a cafeteria diet given during both pregnancy and lactation are far more pronounced [33]. Another caveat of our study is that the DIO offspring were continued on the standard chow. Future studies should evaluate whether DIO offspring weaned onto the obesogenic diet remain glucose intolerant. Indeed, in utero effects are modulated by postnatal nutritional interventions. As an example, the offspring of rats fed a highfat diet during gestation and lactation and weaned onto a highsucrose diet showed a worse metabolic profile than their counterparts weaned onto a standard chow [34]. It would also be interesting to examine the effect of pregnancy in DIO offspring because of the well-documented gestational insulin resistance. For instance, nonfasting glucose concentrations were increased during late gestation in the underweight offspring of severely diabetic rats, whereas these were normal in nongravid age-matched offspring [35]; and the offspring of 
diabetic rats failed to show the normal pregnancy-associated increment in insulin-granulated $\beta$-cells [7].

In summary, our data in a rat model demonstrate that in utero exposure to an environment of energy surfeit and glucose intolerance causes overweight, adipocyte hypertrophy, and increased TNF- $\alpha$ gene expression in WAT in early life (until d35). These changes were no longer observed in postpubertal rats, suggesting that the prenatal effects wane with age.

\section{Acknowledgments}

$\mathrm{SC}, \mathrm{SL}$, and this project were supported by grants from the Fonds voor Wetenschappelijk Onderzoek-Vlaanderen (Belgium), grant G.0221.03, and the Katholieke Universiteit Leuven (OT/02/48).

The authors thank L Vercruysse for her help with the image analysis program, and C Luyten and E Van Herck for their assistance with the analyses.

\section{References}

[1] Pietiläinen KH, Kaprio J, Räsänen M, et al. Tracking of body size from birth to late adolescence: contributions of birth length, birth weight, duration of gestation, parents' body size, and twinship. Am J Epidemiol 2001;154:21-9.

[2] Oken E, Gillman MW. Fetal origins of obesity. Obes Res 2003;11: 496-506.

[3] Silverman BL, Rizzo T, Green OC, et al. Long-term prospective evaluation of offspring of diabetic mothers. Diabetes 1991;40 (Suppl 2):121-5.

[4] Schaefer-Graf UM, Pawliczak J, Passow D, et al. Birth weight and parental BMI predict overweight in children from mothers with gestational diabetes. Diabetes Care 2005;28:1745-50.

[5] Holemans K, Caluwaerts S, Poston L, et al. Diet-induced obesity in the rat: a model for gestational diabetes mellitus. Am J Obstet Gynecol 2004; 190:858-65

[6] Petry CJ, Ozanne SE, Wang CL, et al. Early protein restriction and obesity independently induce hypertension in 1-year-old rats. Clin Sci 1997;93:147-52.

[7] Aerts L, Holemans K, Van Assche FA. Maternal diabetes during pregnancy: consequences for the offspring. Diabetes Metab Rev 1990; 6:147-67.

[8] Caluwaerts S, Holemans $\mathrm{K}$, van Bree R, et al. Is low-dose streptozotocin in rats an adequate model for gestational diabetes mellitus? J Soc Gynecol Invest 2003;10:216-21.

[9] Yamauchi T, Kamon J, Waki H, et al. The mechanisms by which heterozygous peroxisome proliferator-activated receptor $\gamma(\operatorname{PPAR} \gamma)$ deficiency and PPAR $\gamma$ agonist improve insulin resistance. J Biol Chem 2001;276:41245-54

[10] Rodbell M. Metabolism of isolated fat cells. I. Effects of hormones on glucose metabolism and lipolysis. J Biol Chem 1964;239:375-80.

[11] Lambin S, van Bree R, Caluwaerts S, et al. Adipose tissue in offspring of Lepr $^{\mathrm{db} /+}$ mice: early-life environment versus genotype. Am J Physiol Endocrinol Metab 2007;292:E262-71.

[12] Widdowson EM. Chemical composition of newly born mammals Nature 1950;166:626-8.

[13] Bieswal F, Hay SM, McKinnon C, et al. Prenatal protein restriction does not affect the proliferation and differentiation of rat preadipocytes. J Nutr 2004;134:1493-9.

[14] Sypniewska G. Adipogenic activity in the plasma of genetically obese Zucker rats. Acta Physiol Pol 1989;40:520-9
[15] Marques BG, Hausman DB, Latimer AM, et al. Insulin-like growth factor I mediates high-fat diet-induced adipogenesis in OsborneMendel rats. Am J Physiol Regul Integr Comp Physiol 2000;278: R654-62.

[16] Weyer C, Foley JE, Bogardus C, et al. Enlarged subcutaneous abdominal adipocyte size, but not obesity itself, predicts type II diabetes independent of insulin resistance. Diabetologia 2000;43: 1498-506.

[17] Morin CL, Eckel RH, Marcel T, Pagliassotti MJ. High fat diets elevate adipose tissue-derived tumor necrosis factor- $\alpha$ activity. Endocrinology 1997;138:4665-71.

[18] Borst SE, Conover CF. High-fat diet induces increased tissue expression of TNF- $\alpha$. Life Sci 2005;77:2156-65.

[19] Neels JG, Olefsky JM. Inflamed fat: what starts the fire? J Clin Invest 2006; $116: 33-5$.

[20] Lambin S, van Bree R, Vergote I, Verhaeghe J. Chronic tumor necrosis factor(TNF) $-\alpha$ infusion in gravid C57BL6/J mice accelerates adipose tissue development in female offspring. J Soc Gynecol Invest 2006;13: 558-65.

[21] Dahlgren J, Nilsson C, Jennische E, et al. Prenatal cytokine exposure results in obesity and gender-specific programming. Am J Physiol Endocrinol Metab 2001;281:E326-34.

[22] Franks PW, Looker HC, Kobes S, et al. Gestational glucose tolerance and risk of diabetes in young Pima Indian offspring. Diabetes 2006;55: 460-5.

[23] Smith U, Sjöström L, Björntorp P. Comparison of two methods for determining human adipose cell size. J Lipid Res 1972;13: 822-4.

[24] Porter MH, Fine JB, Cutchins AG, et al. Sexual dimorphism in the response of adipose mass and cellularity to graded caloric restriction. Obes Res 2004;12:131-40.

[25] Buckley AJ, Keseru B, Briody J, et al. Altered body composition and metabolism in the male offspring of high fat-fed rats. Metabolism 2005;54:500-7.

[26] Khan IY, Dekou V, Douglas G, et al. A high-fat diet during rat pregnancy or suckling induces cardiovascular dysfunction in adult offspring. Am J Physiol Regul Integr Comp Physiol 2005;288: R127-33.

[27] Rolls BJ, Rowe EA. Pregnancy and lactation in the obese rat: effects on maternal and pup weights. Physiol Behav 1982;28:393-400.

[28] Guo F, Jen K-LC. High-fat feeding during pregnancy and lactation affects offspring metabolism in rats. Physiol Behav 1995;57:681-6.

[29] Siemelink M, Verhoef A, Dormans JAMA, et al. Dietary fatty acid composition during pregnancy and lactation in the rat programs growth and glucose metabolism in the offspring. Diabetologia 2002;45: 1397-403.

[30] West DB, York B. Dietary fat, genetic predisposition, and obesity: lessons from animal models. Am J Clin Nutr 1998;67(Suppl): 505S-12S

[31] Bertin E, Gangnerau M-N, Bailbé D, et al. Glucose metabolism and $\beta$-cell mass in adult offspring of rats protein and/or energy restricted during the last week of pregnancy. Am J Physiol Endocrinol Metab 1999;277:E11-7.

[32] Scaglia L, Cahill CJ, Finegood DT, et al. Apoptosis participates in the remodeling of the endocrine pancreas in the neonatal rat. Endocrinology 1997;138:1736-41.

[33] Bayol SA, Simbi BH, Stickland NC. A maternal cafeteria diet during gestation and lactation promotes adiposity and impairs skeletal muscle development and metabolism in rat offspring at weaning. J Physiol (London) 2005;567:951-61.

[34] Srinivasan M, Katewa SD, Palaniyappan A, et al. Maternal high-fat diet consumption results in fetal malprogramming predisposing to the onset of metabolic syndrome-like phenotype in adulthood. Am J Physiol Endocrinol Metab 2006;291:E792-9.

[35] Holemans K, Aerts L, Van Assche FA. Absence of pregnancy induced alterations in tissue sensitivity in the offspring of diabetic rats. J Endocrinol 1991;131:387-93. 